\section{Der einseitige und verengte Aspekt des Verfassungsgerichts}

* In einer am 3. Januar 1968 beim Bundesarbeitsgericht abgehaltenen Pressekonferenz machte der Präsident des Gerichts, Prof. Dr. Gerhard Müller, erhebliche Bedenken dagegen geltend, daß nach geltendem Recht die Entscheidungen der Obersten Bundesgerichte mit der Verfassungsbeschwerde angefochren werden können. Dies führe zu einer durch die Sache nicht gebotenen Einführung einer Superrevisionsinstanz, zu einer Abwertung des Ansehens der Obersten Bundesgerichte und wegen der langsamen Arbeitsweise des Bundesverfassungsgerichts vielfach zu einer nicht mehr vertretbaren Verzögerung der Erledigung lebenswichtiger Verfahren. Außerdem beurteilte das Bundesverfassungsgericht einschlägige Sachfragen nicht aus der sachkundigen, umfassenden Siche des Obersten Bundesgerichts, sondern vielfach einseitig und verengt aus dem Aspekt des Verfassungsrechts.* (zitiert nach Recht der Arbeit 1968, Heft 2, Seite 57)

Diese Mitteilung verdient vor allem deswegen, hier wiedergegeben zu werden, weil den in ihr verwendeten Argumenten - soweit ersichtlich - bisher nicht öffentlich widersprochen worden ist. Das ist ebenso erschreckend wie die Mitteilung selbst.

Es offenbart ein gestörtes Verhältnis zu der dem Grundgesetz zugrunde liegenden Idee von Demokratie, deren Essentiale die dauernde Kontrolle aller Staatsgewalt ist, wenn behauptet wird, daß das Ansehen einer staatlichen Institution durch das Vorhandensein einer Kontrollinstanz abgewertet wird, ohne daß der Maßstab der Kontrolle und die Legitimation der Kontrollinstanz ins Gewicht fallen. Das - aus dem Blickwinkel der Demokratie zweifelhafte - Ansehen absoluter Monarchen und Diktatoren mag darauf beruhen, $\mathrm{da} B$ sie keiner Kontrolle unterliegen. In der Demokratie kann das Ansehen einer staatlichen Institution nur das Resultat dauerhafter Bewährung gegenüber wirksamer Kontrolle sein. Wo die vom Bundesverfassungsgericht am Maßstab des Grundgesetzes ausgeübre Kontrolle als ansehensmindernd erscheint, ist das Ansehen des Grundgeserzes und des Bundesverfassungsgerichts offenbar von geringerem Interesse als das von Bundesgerichten, die nebenbei von Oberen zu Obersten befördert werden. $\mathrm{Ob}$ das in der Großen Koalition nahezu permanent für Veränderungen zur Disposition gestellte Grundgesetz, dessen insgesamte Ersetzung durch eine andere Verfassung neuerdings allen Ernstes vorgeschlagen wird, durch solche Vorgänge noch an Ansehen verlieren kann, ist allerdings $z$ weifelhaft. Die Tendenz zum Abbau von Kontrollen paßt sich auch in eine politische Entwidklung ein, in der das Ansehen der Bundesregierung ebenfalls immer weniger darauf beruhen kann, daß sie einer wirksamen parlamentarischen Kontrolle standhält. Das mögen die Gründe für das Ausbleiben von Widerspruch sein.

Bei der ins Feld geführten Verzögerung der Erledigung lebenswichtiger Verfahren wird Verfassungsmäßigkeic gegen Beschleunigung ausgespielt, wird das Grundgesetz zur »quantité négligeablex. Das Bundesverfassungsgericht macht von der in $\$ 93$ a Bundesverfassungsgerichtsgesetz vorgesehenen Möglichkeit, offenbar aussichtslose Verfassungsbeschwerden durch den Vorprüfungsausschuß ablehnen zu lassen, regen Gebrauch. Die in den Fällen der Ablehnung eintretende Verzögerung fällt gegenüber der Lauer eines 
durch drei Instanzen geführten Verfahrens nicht ins Gewicht. Wenn eine Verfassungsbeschwerde zugelassen wird, tritt in der Tat eine erhebliche Verzögerung ein. Aber dann ist eine Verletzung des Grundgesetzes immerhin nicht mehr unwahrscheinlich.

Die Gegenüberstellung der »sachkundigen und umfassenden Sicht der Obersten Bundesgerichte" mit dem dem Bundesverfassungsgericht attestierten einseitigen und verengten Aspekt des Verfassungsrechts ist vielleicht nur ein Selbstlob, über dessen guten Geschmads man streiten kann. Es drängt sich aber der Gedanke auf, daß sich hier eine gegen die Kontrolle gerade durch das Bundesverfassungsgerichtgerichtete $A b$ neigung von politischem Inhalt ausdrückt. Nach den Untersuchungen von Feest (Die Bundesrichter, in Zapf, Beiträge zur Analyse der deutschen Oberschicht, München 196s) und den späteren Wahlen neuer Bundesverfassungsrichter kann man annehmen, daß bei der Wahl der Bundesverfassungsrichter mehr als bei den Richtern der Oberen Bundesgerichte Wert auf unzweifelhaft demokratisch geprägte Persönlichkeiten gelegt wird, bei denen zumindest auch niche der Anschein eines ehemals positiven Verhältnisses zum NS-Staat besteht. Auch hat das Erfordernis einer $Z$ weidrittel-Mehrheit bei der Wahl der Bundesverfassungsrichter eine politische Zusammensetzung des Bundesverfassungsgerichts zur Folge, die anders ist als die der Oberen Bundesgerichte, deren Mitglieder mit einfachen Mehrheiten gewählt werden. Das hat das Bundesverfassungsgericht in der Vergangenheit gelegentlich zu einem von dem der Oberen Bundesgerichte abweichenden Verständnis des Grundgeserzes geführt. Wahrscheinlich hat aber in viel zahlreicheren Fällen allein die Möglichkeit der Verfassungsbeschwerde die Oberen Bundesgerictite veranlaßt, bei ihrer Rechtsprechung die von dem so anders gearteten Bundesverfassungsgericht schon aufgestellten oder erwarteten Maßstäbe zu berücksichrigen. So könnte die Möglichkeit der Verfassungsbeschwerde gegen Entscheidungen der Oberen Bundesgerichte auch in Zukunt ein demokratische und rechtsstaatliche Positionen des Grundgesetzes bewahrender Faktor sein. Ein einseiriger und verengter Aspekt?

Jan Geblsen

\section{Bemerkungen \\ zum 8. Strafrechts- änderungsgesetz}

Am 1. 8. 1968 ise als Folge der unaufhörlich gewachsenen Kritik und verstärkter Reformbemühungen das 8 . Strafrechtsänderungsgesetz vom 25.6 . I 968 (BGBl. I, Nr. 43, S. 74I ff.) in Kraft getreten; es bringt unter weirgehender Formulierungshilfe des Bundesjustizministeriums eine neue Fassung des gesamten Hoch- und Landesverrats, sowie Staatsgefährdungsrechts und den Wegfall einzelner sowie die Umformulierung mehrerer Vorschriften, gerade auf dem Gebiete des Staatsschutzes, mit sich.

$\mathrm{Ob}$ das neue Gesetz eine wirkliche $\mathrm{Re}$ form "an Haupt und Gliedern « war, d. b. eine wesentliche Umgestaltung der bisherigen Justizpraxis herbeiführen kann, wird letztendlich erst die $\mathrm{Zu}-$ 